\title{
An improvement of Distance Vector Hop Localization Algorithm for Wireless Sensor Networks
}

\author{
Xiang Yang ${ }^{1}$ \\ School of Information Science and Engineering, Guilin University of Technology \\ Bowen College of Management, Guilin University of Technology \\ School of Information Engineering, Wuhan University of Technology \\ Guilin,541004, China \\ E-mail:nenI vyimo@163.com \\ Meifeng Liu ${ }^{2}$ \\ School of Information Science and Engineering, Guilin University of Technology \\ Guilin ,541004,China \\ E-mail: 785386752 @qq. com
}

\section{Dengteng Deng}

School of Information Science and Engineering, Guilin University of Technology Guilin ,541004 China

E-mail: 1090888216 eqq. com

Due to the un-consideration of random distribution of DV-Hop algorithm in wireless sensor network, a big error caused by using of hop count between unknown and anchor nodes and the nearest anchor nodes' average hop distance instead of the average hop distance of all the anchor nodes. An improved DV-HOP localization algorithm is proposed based on the traditional DVHOP localization algorithm aimed to reduce the localization error in this paper. The proposed algorithm introduce RSSI technique to modify the Hop count of DV-Hop and use the original DV-HOP algorithm to estimate the positions of anchor nodes which are already known, the error between estimation and real distance of anchor nodes so obtained are then to modify the Hop size. The simulation result shows that the proposed algorithm has a better performance in localization precision.

CENet2015

12-13 September 2015

Shanghai, China

\footnotetext{
${ }^{1}$ Speaker

${ }^{2}$ Correspongding Author
} 


\section{Introduction}

Wireless sensor network (WSN) is a large scale ad hoc network with a large number of static or mobile sensor nodes which are random distributed in the monitoring area. The sensors can cooperate with each other to perceive, acquire, process and transmit the information which is perceived in its covered region and then send the information to the owner of the network[12]. So the most significant potential use of sensor nodes in WSN is the location of its own.

Now, the wireless sensor network localization algorithms can be divided into two categories: Range-based and Range-free. The Range based localization techniques including RSSI[3] (received signal strength indicator), TOA(time of arrival), TDOA(time difference on arrival) and AOA(angle of arrival). Amorphous algorithm, APIT algorithm and DV-HOP[4] algorithm are included in the Range free algorithm.

In this paper, we proposed a new algorithm that has combined RSSI and DV-HOP algorithm together to modify the Hop size and Hop count among the nodes based on DV-HOP algorithm. The basic algorithm of RSSI and DV-Hop and some reported improvements are outlined in Section 2. In Section 3, we introduce our proposed algorithm. Results of simulations and their analysis are presented in Section 4. Finally, we conclude the paper in Section 5.

\section{Related work}

\subsection{RSSI and DV-HOP Localization Algorithm}

Received signal strength indicator is a ranging technology according to signal strength. According to the receiver signal strength, distance between sender and receiver can be calculated. Assume that nodes can receive radio signal from node R, RSSI can be calculated through function (2.1).

$$
R_{s}=P_{R} \times K / d_{s}^{\alpha}
$$

Where $R_{s}$ represent RSSI, $P_{R}$ represent emission signal strength, $K$ is a constant, $d_{s}$ represent Euclidean distance and $\alpha$ is changing rate between distance and energy.

DV-HOP algorithm is divided into three stages mainly.

Step 1: In the first stage, anchor nodes using the flooding algorithm broadcast its hops which has initialized of zero and location information to other nodes among the network. The nodes in the network that received the message keep the hops and location information and plus one to the hops to forward it to its neighbor node until the message arrived another anchor node. If the message which the nodes received has come from the same anchor nodes, the nodes will choose one that has minimal hops to record and abandon other packets.

Step 2: Each anchor nodes calculate its Hop size according to function (2.2).

$$
\text { Hopsize }_{i}=\sum_{i \neq j} \sqrt{\left(x_{i}-x_{j}\right)^{2}+\left(y_{i}-y_{j}\right)^{2}} / \sum_{i \neq j} \text { Hopnum }_{i j}
$$

Where $\left(\mathrm{x}_{\mathrm{i}}, \mathrm{y}_{\mathrm{i}}\right)$ and $\left(\mathrm{x}_{\mathrm{j}}, \mathrm{y}_{\mathrm{j}}\right)$ are the coordinates of $\mathrm{i}$ and $\mathrm{j}$ respectively, Hopnum is hop count between anchors $i$ and $j$. Then, each anchor nodes will broadcast the packet including Hopsize information to network again. Unknown nodes save the first packet they receive and then transmit it to neighbors. Lastly, unknown nodes estimate the distance from each anchors by product of Hop count and Hop size.

Step 3: When the unknown nodes receive three or more distance information, it can use either trilateration or maximum likelihood estimation to calculate its coordinates.

\subsection{Review of Improvements to the DV-Hop Localization Algorithm}

Recent years, researchers have proposed many improvements to DV-HOP algorithm.

An union node localization algorithm is put forward to improve localization accuracy, but this algorithm depend largely on the hop count and ignore other ingredients [5]. An improvement is made by the re-estimation of anchor nodes, however, it needs a complex calculation which require a lot of energy consumption [6]. Some investigations have been done on whether the multi-hop communication has enhanced localization accuracy and the author 
proved that using a larger number of hops with a shorter transmission range in dense environments may provide higher accuracy than using a small number of hops with a larger transmission range[7]. Some improvements to the algorithm have been achieved by introducing a weight for the Hop size, but this algorithm can not solve the estimation error of each hop produced by maldistribution of adjacent nodes [8]. An improvement for localization accuracy have been achieved by using the ratio of RSSI to modify hops, however, the algorithms depend largely on the RSSI that will cause accumulate error[9-10].

\section{New DV-HOP Algorithm}

\subsection{Overview}

In this paper, we will use the RSSI algorithm to modify the Hop count and the DV-HOP algorithm to estimate the positions of anchor nodes which are already known, the error between estimation and real distance of anchor nodes so obtained are then to modify the Hop size. The proposed algorithm is divided into the following four steps.

Step 1: Each anchor node generates a packet with a format of $\left\{\mathrm{ID},(x, y)\right.$, Hopnum, $\left.R S S I_{m}, R S S I(m)\right\}$ and broadcast it in a certain power. ID is a unique number and $(x, y)$ is the position coordniate of beacon node, Hopnum is the Hop count has initialized to $0, \mathrm{RSSI}_{\mathrm{m}}$ as the received packet signal strength of the $\mathrm{m}$ hops, where $\mathrm{m}=\operatorname{Hopnum}$. RSSI(m) is cumulative of signal strength from 0 to $\mathrm{m}$ hops. The neighbor nodes will edit the contents of the packet which it received as the following changes where Hopnum=Hopnum +1 and $\operatorname{RSSI}(\mathrm{m})=$ $\mathrm{RSSI}(\mathrm{m}-1)+\mathrm{RSSI}_{\mathrm{m}}$ and forward it to neighbors until the packet arrive another anchor node. If the packet which the nodes received has come from the same anchor nodes, the nodes will choose one that has minimal hops to record and discard other packets.

Step 2: Beacon nodes calculate the average per hop signal strength and Hopsize. Then calculate Estimate and Real to correct Hopsize to figure out Error ${ }_{i j}$ of each Hop size between two beacon nodes. After that, the average error of the total error AverageError ${ }_{i}$ for each hops' Hopsize will be figured out. Lastly, correction coefficient $\alpha_{i}$ for Hopsize will be set up to

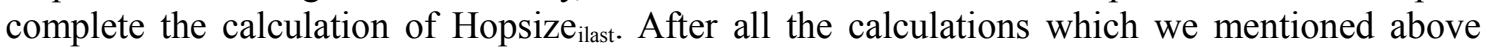
finished, a packet with a format of $\left(I D,\left(x_{i}, y_{i}\right)\right.$, Hopsize $_{\text {ilast }}, R S S I_{\text {avgi }}$, AverageError $\left._{i}, \alpha_{i}\right)$ will be broadcasted by beacon nodes again. When the unknown nodes received the packet, not only the first packet they received will be recorded, but all the packet which has same hops information as the first packet they received, then the packet will be forwarded to neighbors.

Step 3: The AverageRSSI ${ }_{\text {avg }}$ will be figured out from in line with the conditions of RSSI $_{\text {avg }}$ which has received by the unknown nodes first. Then the distance $d$ to beacon nodes will be calculate through function (2.3). Assume that Hopcount from beacon node to unknown node is $\mathrm{h}$, then

$$
d=\left[\left(\alpha_{i} \times \text { Hopsize }\right)+\left(1-\alpha_{i}\right) \times \text { AverageError }_{i}\right] \times\left[R S S I(h) / \text { AverageRSSI }_{\text {avg }}\right]
$$

Step 4: When the unknown nodes receive three or more distance information, it can calculate its coordinates by using either trilateration or maximum likelihood estimation.

\subsection{Hop Count and Hop Size Correction}

In WSN the received signal strength can be obtained directly from wireless node chip register. In this algorithm, RSSI will be used to estimate hops.

In the first stage of new DV-HOP algorithm, the packets which forward by beacon nodes are including the message of RSSI from the last hop and cumulative RSSI from first hop. When the broadcasting message arrive a node, the node will recorded cumulative RSSI and only the one which has the least hops will be recorded. Then, the average per hop signal strength RSSI avgi $_{\text {ing }}$ will calculate through function (3.1). 


$$
R S S I_{\text {avgi }}=\sum_{n=1}^{w} \operatorname{RSSI}\left(q_{n}\right) / \sum_{n=1}^{w} q_{n}
$$

Where $\mathrm{n}$ is the amount of beacon nodes in the communication range of node $\mathrm{i}, \mathrm{q}_{\mathrm{n}}$ is the least hops to other beacon nodes. For the second broadcasting, RSSI $_{\text {avgi }}$ will be broadcasted by beacon nodes. Unknown nodes will receive and record the packet message which has same hops information as the first packet they received. Then, each unknown node will figure out AverageRSSI ${ }_{\text {avgj }}$ through function (3.2).

$$
\text { AverageRSSI } I_{\text {avg }}=\sum_{l=1}^{b} R S S I_{\text {avgi }} / b
$$

Where $b$ is the number of beacon nodes that has same hops. After the broadcasting completed, the Hopnum between unknown and beacon nodes can be calculated through function (3.3).

$$
\text { Hopnum }=\operatorname{RSSI}(m) / \text { AverageRSSI }{ }_{\text {avg }}
$$

Where $\operatorname{RSSI}(\mathrm{m})$ is the cumulative of signal strength from beacon node to unknown node.

In new DV-Hop algorithm, we will use Hopszie to estimate distance Estimate between beacon nodes. As is shown in function (3.4), the Estimate can be figure out.

$$
\text { Estimate }=\text { Hopszie } \times \text { Hopcount }
$$

Where Hopcount is hops between two beacon nodes. At the same time, the beacon nodes calculate the real distance Real to other beacon nodes through function (3.5).

$$
\operatorname{Re} a l=\sqrt{\left(x_{i}-x_{j}\right)^{2}+\left(y_{i}-y_{j}\right)^{2}}
$$

Then, the Error ${ }_{i j}$ of per jump distance will be figure out through function (3.6).

$$
\text { Error }_{i j}=\mid \operatorname{Re} a l-\text { Estimate } \mid / q
$$

Where $\mathrm{q}$ is hopcount between two beacon nodes. Next, AverageError ${ }_{i}$ and correction coefficient $\alpha_{\mathrm{i}}$ can be figured out through functions (3.7) and (3.8).

$$
\begin{array}{r}
\text { AverageError }_{i}=\sum_{j=1}^{n} \text { Error }_{i j} / n \\
\left.\alpha_{i}=\text { Hopsize }_{\text {Hopsize }}+\text { AverageError }_{i}\right)
\end{array}
$$

After all the calculation we mentioned above finished, Hopsize $\mathrm{i}_{\text {ilast }}$ will be figured out through function (3.9).

$$
\text { Hopsize }_{\text {ilast }}=\left(\alpha_{i} \times \text { Hopsize }\right)+\left[\left(1-\alpha_{i}\right) \times \text { AverageError }_{i}\right]
$$

\section{Simulation Results and Discussion}

In order to evaluate the performance of the improved algorithm, the improved algorithm, referenced algorithm and DV-HOP algorithm were simulated by Matlab. The simulation parameter setting is that in the square of $100 \times 100 \mathrm{~m}$ randomly deployed 100 sensor nodes. Through simulation, the beacon nodes proportion and communication radius influence on localization accuracy will be studied. All the simulation results are averaged over 100 runs. Localization error defined as follows

$$
e_{i}=\frac{\sqrt{\left(x_{i_{-} \text {real }}-x_{i}\right)^{2}+\left(y_{i_{-} \text {real }}-y_{i}\right)^{2}}}{R} \quad 100 \%
$$

Where $\left(\mathrm{x}_{\mathrm{i}}, \mathrm{y}_{\mathrm{i}}\right)$ and $\left(\mathrm{x}_{\mathrm{i}_{-} \text {real }}, \mathrm{y}_{\mathrm{i}_{-} \text {real }}\right)$ are the estimated and real coordinates of the node $\mathrm{i}$ and $\mathrm{R}$ is the communication radius.

Figure 1 shows the simulation results of original DV-Hop algorithm, new DV-Hop algorithm and referenced algorithm with communication radius 40 meter, beacon node ratio changes from 10 percent to 50 percent. 


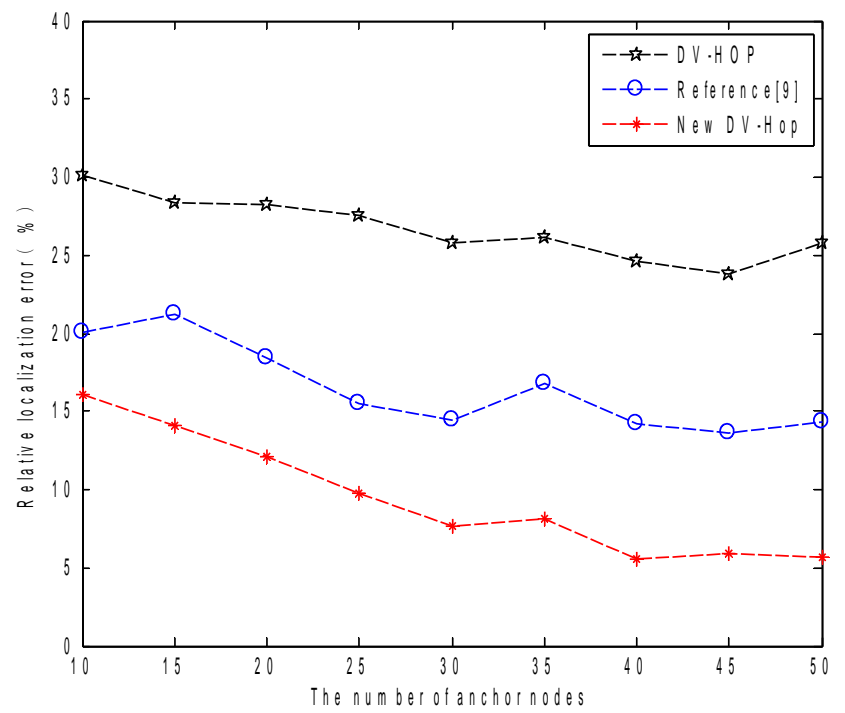

Figure 1: Relative position error comparison chart with $\mathrm{R}=40 \mathrm{~m}$

From the figure we can know that with the proportion increase of beacon nodes, the relative localization error gradually reduced, but the improved algorithm reduced more significant, especially when the beacon node ratio changes from 10 percent to 30 percent. Compared with the original DV-Hop and referenced algorithm, the localization error of the proposed algorithm reduced about $20 \%$ and $8 \%$ respectively. That is because that with the increase of anchor nodes, the connectivity of networks will promote, so the neighbor nodes of unknown node will increase. With that the unknown nodes will immediately acquire more AverageRSSI $\mathrm{avg}_{\text {ag }}$ which used to modify the hops to diminish the error and the Hopsize ilast $_{\text {will be }}$ modified more accurate, since the more anchor nodes the least hop counts among the anchor nodes and the re-estimation of Hopsize will be more precise.

Figure 2 shows the simulation results of original DV-Hop algorithm, new DV-Hop algorithm and referenced algorithm with beacon node ratio is about $30 \%$, communication radius changes from 20 to 50 meters.

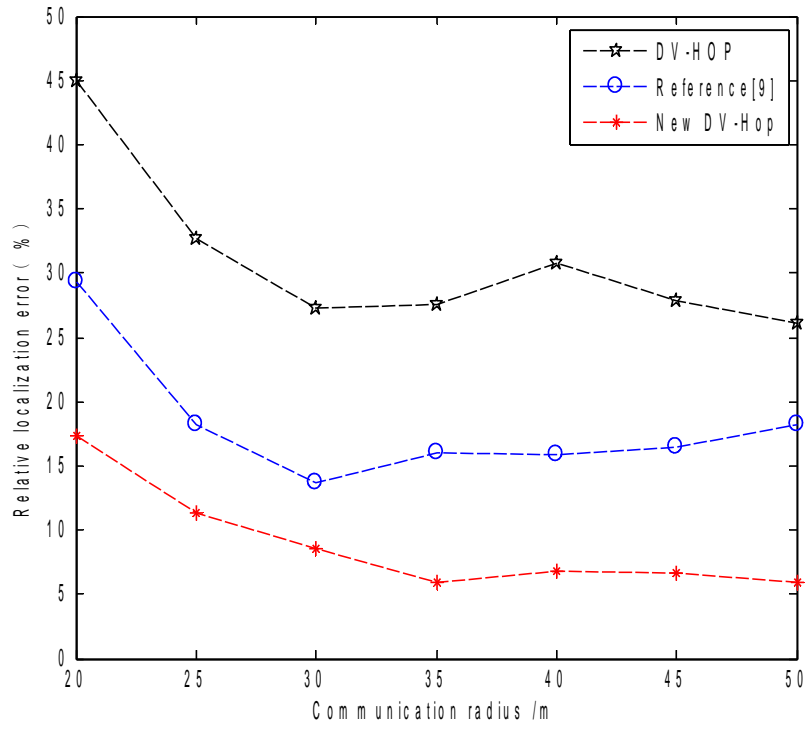

Figure 2: Relative localization error comparison chart with Beacon nodes $=30$ 
From the figure we can know that the relative positioning error decreases gradually with the increase of communication radius. In these three algorithms, the proposed algorithm has the lowest localization error. For instance, when the communication radius is 35 meters, the relative localization error of original DV-Hop is 27.5368, referenced algorithm is 15.9974 and the proposed algorithm is 5.9682 . That is because with the increase of communication radius, the connectivity of the network will be lifted and the unknown node can communicate with more anchor nodes to acquire more accurate hops to diminish the error which accumulated by multiple hops.

Figure 3 shows the simulation results of proposed DV-Hop algorithm with communication radius are $30,40,50$ and 60 meters and beacon node ratio changes from $10 \%$ to $50 \%$.

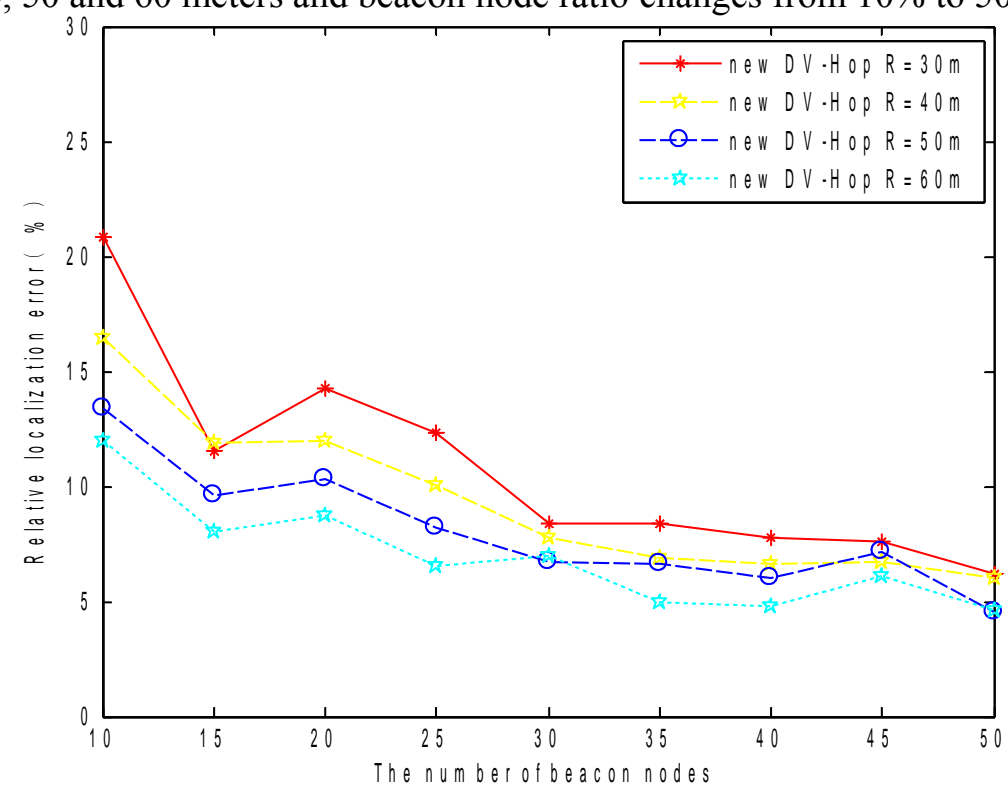

Figure 3: Relative localization error comparison chart of proposed DV-Hop algorithm with different radius

From the figure we can know that the relative localization error decreases with the increase of communication radius. With the increase of anchor nodes the localization error of same communication radius will reduce either. That is because that with the increase of communication radius and anchor nodes, the network connectivity will be lifted obvious. This simulation result is corresponding with simulation results of Fig 1 and Fig 2.

\section{Conclusion}

This paper put forward an improved DV-HOP algorithm based on Hop size correction through re-estimation of anchor nodes and hops modified through the ratio of RSSI. Compared with other localization algorithm, this algorithm has the advantages of high localization accuracy and does not require additional hardware support, for different node distribution also keeps high performance. However, this algorithm has some disadvantages as well, since the new improved localization algorithm increases the traffic information transmission and the extra relocation computational cost of beacon node.

\section{References}

[1] Jennifer Yick, Biswannath Mukherjee and Dipak Ghosal. Wireless sensor network survey [J]. Computer Networks. 52 (12):2292-2230(2008)

[2] Zhihong Qian, Yijun Wang. Internet of Things-Oriented Wireless Sensor Networks Review[J]. Journal of Electronics\&Information Technology. 35(1): 215-227(2013)(In Chinese) 
[3] Wei Wang. Attack Security and Defense Technology of Sybil in Wireless Sensor Networks[M]. National Defense Industry Press. Beijing. 70-71(2013)(In Chinese)

[4] Liping Xiao, Xiaohong Liu. DV-HOP localization algorithm based on hops modify[J]. Chinese Journal of Sensors and Actuators. 25(12):1726-1630(2012)(In Chinese)

[5] Dengyin Zhang, Guodong Cui. A union node localization algorithm based on RSSI and DV-HOP for WSNs [C] //Instrumentation, Measurement, Computer, Communication and Control (IMCCC), 2012 Second International Conference on. IEEE. Ha erbin 1094-1098(2012)

[6] Samiru Gayan, PWiMob. Improved DV-HOP Algorithm Through Anchor Position Reestimation[C]// 2014, IEEE, Bali 28-30 Augustus 2014 Dileeka Dias. 126-131(2014)

[7] Lixia Wang, Pai Zhang. A New DV-HOP Algorithm in Wireless Sensor Network[C] //2013 2nd International Symposium on Instrumentation and Measurement, Sensor Network and Automation (IMSNA). IEEE.Georgetown.194-197(2013)

[8] Jian Li, Jianmin Zhang and Xiande Liu. A weighted dv-hop localization scheme for wireless sensor networks $[\mathrm{C}]$. In Scalable Computing and Communications, Eighth International Conference on Embedded Computing, 2009.IEEE. Dalian. 269-272(2009)

[9] Xiang Yang, Wei Pan. DV-Hop localization algorithm for wireless sensor networks based on RSSI ratio improving [J]. Transducer and Microsystem Technologies. 32(7):126-128(2012)(In Chinese)

[10] Xiaobo Zhou, Gangzhu Qiao and Jianchao Zeng. RSSI based weighted DV-HOP localization algorithm for Wireless Sensor Networks Networks[J]. Computer Engineering and Applications. 47(14):109-111(2011)( In Chinese) 